wild-type mice. By contrast, IL-17deficient mice and $\gamma \delta$ T cell-deficient mice showed normal long-term spatial reference memory as assessed by Morris water maze tests.

The authors found that IL-17 supports long-term potentiation (LTP) and the plasticity of glutamatergic synapses during short-term learning but is not essential for long-term memory training. They identified glial cells as the likely target of IL-17 and showed that IL-17 stimulates glial cells to secrete brain-derived neurotrophic factor (BDNF), which has previously been shown to support synaptic transmission and LTP. Importantly, intracerebroventricular injection of BDNF restored short-term working memory in IL-17-deficient mice.

This study indicates yet another important physiological role for IL-17-producing $\gamma \delta$ T cells and furthers our understanding of how immune mediators affect cognition.

Yvonne Bordon

ORIGINAL ARTICLE Ribeiro, M. et al. Meningea $\gamma \delta$ T cell-derived IL-17 controls synaptic plasticity and short-term memory. Sci. Immunol. 4, eaay5199 (2019)

immunosuppressed patients. The reduction was more pronounced in immunocompetent patients, suggesting that $\mathrm{CD} 8^{+} \mathrm{T}$ cells can target transformed keratinocytes containing active $\beta$-HPV virus and that this may boost immunity against transformed cells. The authors further showed that peptides derived from $\beta$-HPV (but not from a high-risk HPV strain) activated $\mathrm{CD}^{+} \mathrm{T}$ cells from the normal facial skin of immunocompetent adults.

These findings suggest that colonization with commensal $\beta$-HPVs helps to prime $C D 8^{+} \mathrm{T}$ cells in the healthy skin; these tissue-resident $\mathrm{CD}^{+} \mathrm{T}$ cells can then target

keratinocytes that contain active virus while they are undergoing abnormal proliferation to form a wart or a skin cancer. Therefore, far from being a hit and run culprit in skin cancer, $\beta$-HPV seems to in fact be a key ally of $C D 8^{+} T$ cells in mediating immunity against cancer.

Yvonne Bordon

ORIGINAL ARTICLE Strickley, J. D. et al. Immunity to commensal papillomaviruses protects against skin cancer. Nature https:// doi.org/10.1038/s41586-019-1719-9 (2019)

\title{
Commensal viruses contribute to gut health
}

Whereas numerous studies have investigated the role of commensal bacteria in mucosal homeostasis, it is easy to forget that the gut microbiome also contains fungi and viruses. Previous work has associated the dysbiosis of commensal viruses with inflammatory bowel diseases, but the underlying mechanism was not known. Reporting in Nature Immunology, Liu et al. describe a role for commensal viruses in maintaining intraepithelial lymphocytes (IELs) through the retinoic acid inducible gene I (RIG-I)-dependent production of IL-15.

These authors used an antiviral cocktail (AVC) to deplete commensal viruses in the gut of C57BL/6 J wild-type mice. After 6 weeks of treatment, starting at 2 weeks after birth, the number of faecal virus-like particles (from both DNA and RNA viruses) was reduced markedly. AVC-treated mice had decreased numbers of IELs in the small intestine and colon, in particular CD $8 \alpha \alpha^{+}$TCR $\alpha \beta^{+}$and CD $8 \alpha \beta^{+}$TCR $\alpha \beta^{+}$IELs. There were no differences between AVC-treated and control mice in terms of lymphocyte numbers in other organs or percentages of the major immune cell subpopulations. AVC treatment further reduced the number of IELs in antibiotic-treated mice, which suggests that the effect involves eukaryotic viruses and is not owing only to bacteriophage depletion.

Further studies showed that virus recognition for IEL maintenance depends on RIG-I, but not other virus-sensing receptors. Dd $\times 58^{-/-}$mice (which lack RIG-I) and mice deficient for mitochondrial antiviral signalling protein (MAVS; the adaptor protein for RIG-I) had a similar phenotype to AVCtreated mice, and AVC treatment of Dd $\times 58^{-/-}$mice had no further impact on IEL numbers. Intraperitoneal supplementation with a RIG-I ligand rescued the IEL loss in AVC-treated $D d \times 58^{+/+}$mice but not in $D d \times 58^{-/-}$mice. Therefore, commensal viruses support IEL numbers through RIG-I-MAVS signalling. Mice with defective RIG-I-MAVS signalling had normal numbers of thymic precursors for IELs but lower levels of IEL proliferation and higher levels of IEL apoptosis. Thus, RIG-I-MAVS signalling regulates IEL homeostasis rather than development. Although AVC treatment and Ddx58 knockout can also result in bacterial dysbiosis, the authors used co-housing experiments to rule this out as a cause of IEL loss.

A series of adoptive bone-marrow transfer experiments showed that IEL-extrinsic RIG-I signalling in haematopoietic cells is required for IEL homeostasis. Conditional knockout of $D d \times 58$ in CD11c dendritic cells (DCs) and macrophages resulted in decreased numbers of CD $8 \alpha \alpha^{+}$TCR $\alpha \beta^{+}$and CD $8 \alpha \beta^{+}$TCR $\alpha \beta^{+}$IELs, which indicates a crucial role for RIG-I signalling in antigen-presenting cells (APCs). RIG-I signalling had no effect on the development of intestinal macrophages and DCs. Rather, APCs from Ddx58 AVC-treated mice expressed much lower levels of IL-15 than wild-type and control-treated APCs. Furthermore, viral vector-mediated gene delivery of $/ 115$ to $\mathrm{Dd} \times 58^{-/-}$mice and AVC-treated mice restored their numbers of IELs. Together, the results indicate that APC-derived IL-15 maintains IELs in response to commensal virus-induced RIG-I-MAVS signalling. Furthermore, the transcription factor IRF1 was shown to have a crucial role in IL-15 induction mediated through this pathway.

IELs have well-described roles in homeostasis and host defence of the gut mucosa. In keeping with this, both AVC-treated and $\mathrm{Mavs}^{-1-}$ mice were more susceptible than control-treated and wild-type mice to dextran sulfate sodium (DSS)-induced colitis; this susceptibility could be reduced by 1115 gene delivery to restore IEL numbers.

In summary, this study indicates that commensal viruses have similar, but independent, physiological roles to commensal bacteria in maintaining intestinal homeostasis and thus should not be overlooked in studies of intestinal health and disease.

Kirsty Minton

ORIGINAL ARTICLE Liu, L. et al. Commensal viruses maintain intestinal intraepithelial lymphocytes via noncanonical RIG-I signaling. Nat. Immunol. https://doi.org/10.1038/s41590-019-0513-z (2019) 